\title{
Localization of wave fields in lower hybrid cavities
}

\author{
A. Tjulin ${ }^{1,2, *}$, A. I. Eriksson ${ }^{1,2}$, and M. André ${ }^{1,2}$ \\ ${ }^{1}$ Swedish Institute of Space Physics, Uppsala, Sweden \\ ${ }^{2}$ Department of Astronomy and Space Physics, Uppsala University, Sweden \\ *now at: LPCE/CNRS, 3A, avenue de la Recherche Scientifique 45071 Orléans, France
}

Received: 1 August 2003 - Revised: 5 March 2004 - Accepted: 5 May 2004 - Published: 7 September 2004

\begin{abstract}
We investigate lower hybrid wave trapping in cylindrically symmetric density depletions in the electrostatic approximation. Our investigation is inspired by previous observations of such trapping by spacecraft in the auroral region at altitudes up to about $2000 \mathrm{~km}$, and the recent discovery of this phenomenon at altitudes above $20000 \mathrm{~km}$ in the inner magnetosphere. No particular shape is assumed for the density depletion, which need not be strictly zero outside some value of the radial coordinate $r$. Important previously known properties concerning parabolic density depletions extending to finite $r$ are shown to hold also for arbitrary shapes and infinite extent: for a given parallel wave number $k_{z}$, modes below the ambient lower hybrid frequency $f_{\mathrm{LH}}$ are trapped in the density depletion (in the sense that they are evanescent outside the cavity), have a discrete spectrum and rotate in a left-handed sense, while there is a continuous spectrum of freely propagating right-handed rotating modes above $f_{\mathrm{LH}}$. New results are such that even though the density depletion may go to zero slowly with increasing $r$, and thus be essentially infinite in extent, there is a maximum distance within which a trapped mode with given $k_{z}$ and azimuthal mode number $m$ may propagate. Furthermore, we find that for any monotonic density cavity and given $k_{z}$, there is a local relation between plasma density gradient and the lowest possible frequency that can be trapped. We combine our theoretical results with spacecraft observations to find an upper bound on $k_{z}$. Our examples indicate that the length of the cavities is larger than the width by a factor of at least 100 .
\end{abstract}

Key words. Electromagnetics (general or miscellaneous) Magnetospheric physics (plasma waves and instabilities) Space plasma phusics (waves and instabilities)

\section{Introduction}

A Lower Hybrid Cavity (LHC) is a localized density depletion with an increased amplitude of waves in the lower hybrid frequency range. The same phenomenon is also often called a Lower Hybrid Solitary Structure. From observations, the

Correspondence to: A. Tjulin

(Anders.Tjulin@cnrs-orleans.fr) width of the cavity is a few to a few tens of the ambient ion gyroradius, and it has a quasi-cylindrical geometry with the size parallel to the geomagnetic field much bigger than the perpendicular size, though the parallel extension is less well known than the perpendicular. Observed density depletions ranges from $0.1 \%$ to $90 \%$. LHCs have been observed in the ionosphere by sounding rockets (e.g. Vago et al., 1992; Pinçon et al., 1997; Lynch et al., 1999; Knudsen et al., 1999), and the Freja satellite (e.g. Eriksson et al., 1994; Pécseli et al., 1996; Dovner et al., 1997; Høymork et al., 2000) at altitudes up to about $2000 \mathrm{~km}$. Recently, LHCs have been identified further out in the magnetosphere in the Viking and Cluster data sets (Tjulin et al., 2003) at altitudes up to above $20000 \mathrm{~km}$. A recent review of observational and theoretical work on LHCs has been provided by Schuck et al. (2003).

Initial theoretical work attempted to model LHCs as dynamical structures driven by the modulational instability or other nonlinear collapse mechanisms. However, statistical investigations (e.g. Pécseli et al., 1996; Kjus et al., 1998; Schuck et al., 2002) have shown that the LHCs observed on spacecraft are essentially non-transient at least on the time scale of the observation, which is on the order of $10 \mathrm{~ms}$ to $100 \mathrm{~ms}$ depending on the spacecraft. This puts the stationary, as opposed to dynamical, properties of LHCs into focus. Seyler (1994) introduced a model of LHCs as twodimensional cavity modes in a finite cavity of parabolic shape, with the boundary condition of vanishing electric field at infinity. Schuck et al. (1998) described the wave field in LHCs in terms of electrostatic cavity modes in a finite parabolic density depletion, but included a parallel wave number and considered the scattering problem with propagating waves at infinity. This treatment showed a continuous spectrum of right-hand rotating modes above the ambient lower hybrid frequency $f_{\mathrm{LH}}$ and a discrete spectrum of trapped left-hand rotating waves below $f_{\mathrm{LH}}$, that cannot propagate outside the depletion. The results very well describe the properties of LHCs observed on sounding rockets (Pinçon et al., 1997; Bonnell et al., 1998; Schuck et al., 1998) and also of a Cluster satellite observation (Tjulin et al., 2003) for the single event for which data rates allowed such detailed analysis. 
Calculations have mostly been performed in the electrostatic approximation, with some justification from the cavity width being well below the electron inertial length $c / \omega_{\text {pe }}$. Even so, it is clear that electromagnetic effects are of interest, when studying oscillations below $f_{\mathrm{LH}}$, as there is no electrostatic mode in this frequency range to couple to. Though the original equations and numerical examples by Seyler (1994) included a simplified treatment of wave magnetic fields, little has been done on this until the recent work by Hall et al. (2004), who succeeded in developing a cold electromagnetic theory for cylindrically symmetric density depletions, and thus were able to model the coupling of LHC modes to magnetosonic waves propagating outside the cavity.

Thermal effects could also become important close to the lower hybrid frequency, as can be seen from the warm plasma dispersion relation for lower hybrid waves by Shapiro et al. (1993),

$\omega=\omega_{\mathrm{LH}}\left(1+\frac{1}{2} k^{2} R^{2}+\frac{m_{\mathrm{i}}}{2 m_{\mathrm{e}}} \frac{k_{z}^{2}}{k_{\perp}^{2}}\right)$,

where the parallel wave number much exceeds the perpendicular, i.e. $k_{z} \ll k_{\perp}, \omega_{\mathrm{LH}}$ is the lower hybrid resonance angular frequency in the plasma outside the cavity, $m_{\mathrm{i}}$ and $m_{\mathrm{e}}$ are the ion and electron masses, and the thermal dispersion length is

$R=\sqrt{\frac{3 T_{\mathrm{i}}}{\omega_{\mathrm{LH}}^{2} m_{\mathrm{i}}}+\frac{2 T_{\mathrm{e}}}{\omega_{\mathrm{ce}}^{2} m_{\mathrm{e}}} \frac{\omega_{\mathrm{pe}}^{2}}{\omega_{\mathrm{pe}}^{2}+\omega_{\mathrm{ce}}^{2}},}$

with $\omega_{\text {pe }}$ and $\omega_{\text {ce }}$ having their usual meanings of electron plasma and cyclotron angular frequencies, respectively. In the cold plasma case we have $R=0$, so in this case lower hybrid waves are non-propagating in the limit of vanishing $k_{z}$. This suggests that thermal effects could also be important for LHCs, at least for frequencies close to the local $\omega_{\mathrm{LH}}$. However, the trapped modes in LHCs are usually observed well below this frequency. Furthermore, Schuck and Bonnell (2003) have demonstrated in a ray-tracing study that the Hall effect on the electron motion in a density gradient is much more important at least in the WKB approximation.

It is clear that extensions of present cold electrostatic theory for LHC cavity modes to also include thermal and electromagnetic effects without sacrificing the Hall term are needed. The considerations above, however, show that it should still be worthwhile to further explore the cold electrostatic theory introduced by Schuck et al. (1998). An attractive feature of this model is that it leads to a single Bessellike linear ordinary differential equation, which is very suitable for model studies. In the following, we will within this model investigate some properties of this equation for a general density profile, particularly addressing the questions of localization of wave fields. How well are trapped modes confined in a general density cavity? How does the results obtained for parabolic profiles apply to other profiles, for example, the Gaussian profiles reported from observations by Høymork et al. (2000)? What can be said in general terms about the frequency and wave number of trapped modes and their relation to the depth and gradients of density cavities? And can we find a way to estimate the parallel wave number, $k_{z}$, using spacecraft observations from perpendicular crossings of LHCs?

\section{The basic equations}

The waves we are interested in are in the lower hybrid frequency range. In the plasma regions we will consider here, where $\omega_{\mathrm{ci}} \lesssim \omega_{\mathrm{pi}}$, this means that the angular frequencies $\omega$ satisfy the condition $\omega_{\mathrm{ci}} \ll \omega \ll \omega_{\mathrm{ce}}$, where $\omega_{\mathrm{ci}}$ is the ion cyclotron frequency and $\omega_{\mathrm{pi}}$ the ion plasma frequency. This condition means that the ions can be considered unmagnetized and the electrons fully magnetized. Following the derivation of Schuck et al. (1998) for a cold plasma with electrostatic linear perturbations we arrive at the following set of equations

$$
\begin{aligned}
& \frac{\partial n_{\mathrm{i}}}{\partial t}=-n_{0} \nabla_{\perp}^{2} \chi \\
& \frac{\partial \chi}{\partial t}=-\frac{e}{m_{\mathrm{i}}} \varphi \\
& \frac{\partial \Gamma}{\partial t}-n_{0} \frac{\nabla_{\perp} \varphi \times \hat{z}}{B_{0}} \cdot \nabla_{\perp} v=-n_{0} \frac{\partial v}{\partial z} \\
& \frac{\partial v}{\partial t}=\frac{e}{m_{\mathrm{e}}} \frac{\partial \varphi}{\partial z} \\
& \nabla_{\perp}^{2} \varphi=-\frac{e}{\varepsilon_{0}}\left(n_{\mathrm{i}}-n_{\mathrm{e}}\right) \\
& \Gamma=n_{\mathrm{e}}+\frac{n_{0} m_{\mathrm{e}}}{e B_{0}^{2}} \nabla_{\perp}^{2} \varphi,
\end{aligned}
$$

where we have assumed the background magnetic field $\boldsymbol{B}_{0}$ to be in the $\hat{z}$-direction, and that the gradients along the magnetic field are negligible compared to the perpendicular gradients. To include effects from small inhomogeneities in the background plasma density, the parameter $v$ has been included, so that the background density is given by $n_{0}(1+v)$ and $n_{0}$ is the plasma number density far away from any fluctuations.

Equation (4) is the momentum equation for the ions, where $\varphi$ is the electrostatic potential. The ion motion is here assumed to depend only on the electric forces. Equation (3) is the continuity equation for the ions, where $n_{\mathrm{i}}$ is the ion number density. We have also introduced the velocity potential $\chi$, which gives the ion velocity $\boldsymbol{u}_{\mathrm{i}}=\nabla \chi$. It is possible to introduce this since we are only considering electrostatic perturbations and the ions are unmagnetized. The motion of the ions is thus only dependent on the electrostatic potential $\varphi$, which makes this simplification valid. We have in Eq. (3) also assumed that the scale length for the variation of the density cavity is much larger than the scale length of the plasma wave perturbations.

Equation (5) is the electron continuity equation, under the assumption that the electrons are fully magnetized, and hence 
their motion is given by their gyro-center drift motion. The parameter $\Gamma$, defined in Eq. (8), is the electron density, $n_{\mathrm{e}}$, modified for the inclusion of polarization drift corrections, and $v$ is the electron velocity along the magnetic field. Equation (6) is the momentum equation for the electrons along the magnetic field, and Eq. (7) is Poisson's Equation that couples the dynamics of the ions and the electrons. We have here a system of six equations with six unknowns.

\section{The density cavity and rotating wave modes}

We may now introduce a background density variation. We assume that the density depletion is cylindrically symmetric, and that the perturbed quantities are of the form

$$
f(r, \theta, z, t)=\tilde{f}(r) e^{i\left(m \theta+k_{z} z-\omega t\right)},
$$

where $m$ is an integer. This is basically an assumption that the solutions of the system of equations are rotating with respect to the magnetic field. The sense of rotation for these wave solutions is given by the relative sign between $\omega$ and $m$. We may here assume $m$ to be positive, then positive $\omega$ means a right-handed rotation with respect to the magnetic field and negative $\omega$ means a left-handed rotation. The system of Eqs. (3-8) can, under these assumptions, be written as

$$
\begin{aligned}
& -i \omega n_{\mathrm{i}}=-n_{0} \tilde{\nabla}_{\perp}^{2} \chi \\
& -i \omega \chi=-\frac{e}{m_{\mathrm{i}}} \varphi \\
& -i \omega \Gamma-\frac{i m n_{0}}{B_{0} r} \frac{d v}{d r} \varphi=-i n_{0} k_{z} v \\
& -i \omega v=\frac{i e k_{z}}{m_{e}} \varphi \\
& \tilde{\nabla}_{\perp}^{2} \varphi=-\frac{e}{\varepsilon_{0}}\left(n_{\mathrm{i}}-n_{\mathrm{e}}\right) \\
& \Gamma=n_{\mathrm{e}}+\frac{n_{0} m_{\mathrm{e}}}{e B_{0}^{2}} \tilde{\nabla}_{\perp}^{2} \varphi,
\end{aligned}
$$

where we have introduced

$\tilde{\nabla}_{\perp}^{2}=\frac{1}{r} \frac{\partial}{\partial r}\left(r \frac{\partial}{\partial r}\right)-\frac{m^{2}}{r^{2}}$,

and used the cylindrically symmetric density depletion $v(r)$.

Now we can find an expression for how the ion density $n_{\mathrm{i}}$ depends on the electrostatic potential $\varphi$ by combining Eqs. (10) and (11)

$n_{\mathrm{i}}=-\frac{n_{0} e}{m_{\mathrm{i}} \omega^{2}} \tilde{\nabla}_{\perp}^{2} \varphi$.

We may also write $\Gamma$ in terms of the electrostatic potential, $\varphi$, by combining Eqs. (12) and (13)

$\Gamma=-\left(\frac{n_{0} e k_{z}^{2}}{m_{\mathrm{e}} \omega^{2}}+\frac{m n_{0}}{B_{0} r \omega} \frac{d v}{d r}\right) \varphi$, and thus from Eq. (15) the electron density, $n_{\mathrm{e}}$, is given by

$n_{\mathrm{e}}=-\left(\frac{n_{0} e k_{z}^{2}}{m_{\mathrm{e}} \omega^{2}}+\frac{m n_{0}}{B_{0} r \omega} \frac{d \nu}{d r}+\frac{n_{0} m_{\mathrm{e}}}{e B_{0}^{2}} \tilde{\nabla}_{\perp}^{2}\right) \varphi$.

Inserting our expressions for $n_{\mathrm{i}}$ and $n_{\mathrm{e}}$, Eqs. (19) and (17), into Eq. (14) and dividing by $e^{i\left(m \theta+k_{z} z-\omega t\right)}$, we obtain the equation

$$
\begin{aligned}
(1 & \left.-\frac{n_{0} e^{2}}{\varepsilon_{0} m_{\mathrm{i}} \omega^{2}}+\frac{n_{0} m_{\mathrm{e}}}{\varepsilon_{0} B_{0}^{2}}\right) \tilde{\nabla}_{\perp}^{2} \tilde{\varphi} \\
& +\left(\frac{n_{0} e^{2} k_{z}^{2}}{\varepsilon_{0} m_{\mathrm{e}} \omega^{2}}+\frac{m n_{0} e}{\varepsilon_{0} B_{0} r \omega} \frac{d \nu}{d r}\right) \tilde{\varphi}=0
\end{aligned}
$$

for the radial part, $\tilde{\varphi}(r)$, of the electrostatic potential. We may now use the definition of the plasma frequency, and the lower hybrid frequency $\omega_{\mathrm{LH}}$, which in the plasma regions we are interested in is given by

$\omega_{L H}^{2}=\frac{\omega_{\mathrm{ce}}^{2} \omega_{\mathrm{pi}}^{2}}{\omega_{\mathrm{ce}}^{2}+\omega_{\mathrm{pe}}^{2}}$,

to rewrite Eq. (20) as

$$
\frac{\omega_{\mathrm{pi}}^{2}\left(\omega^{2}-\omega_{L H}^{2}\right)}{\omega_{L H}^{2}} \tilde{\nabla}_{\perp}^{2} \tilde{\varphi}+\omega_{\mathrm{pe}}^{2}\left(k_{z}^{2}+\frac{m \omega}{\omega_{\mathrm{ce}} r} \frac{d \nu}{d r}\right) \tilde{\varphi}=0 .
$$

In the following we will use $\omega_{\mathrm{LH}}$ to denote the lower hybrid frequency far from the cavity, and not the local value of the lower hybrid frequency, which varies as the density varies in the cavity.

To make Eq. (22) more compact, we can introduce a new function $A(r)$, defined by

$A(r)=\frac{m_{\mathrm{i}} \omega_{L H}^{2}}{m_{\mathrm{e}}\left(\omega^{2}-\omega_{L H}^{2}\right)}\left(k_{z}^{2}+\frac{m \omega}{\omega_{\mathrm{ce}} r} \frac{d v}{d r}\right)$,

which makes it possible for us to write Eq. (22) as

$\frac{d^{2} \tilde{\varphi}}{d r^{2}}+\frac{1}{r} \frac{d \tilde{\varphi}}{d r}+\left(A(r)-\frac{m^{2}}{r^{2}}\right) \tilde{\varphi}=0$,

using the definition of $\tilde{\nabla}_{\perp}^{2}$ found in Eq. (16). If the density cavity is given by $v(r)=C r^{2}+D$, the function $A(r)$ turns into a constant and Eq. (24) becomes Bessel's equation, for which the solutions are well known (Watson, 1944). This special case has been investigated by Schuck et al. (1998), where the density profile was given by

$v(r)=\left\{\begin{array}{cc}-\Delta\left(1-\frac{r^{2}}{a^{2}}\right), & r<a \\ 0, & r>a\end{array}\right.$.

The result was that inside the cavity the waves were rotating in a right-handed direction about the magnetic field at frequencies above the ambient lower hybrid frequency, and in the left-handed direction below $\omega_{\mathrm{LH}}$. Outside the cavities, all waves had frequencies above the lower hybrid frequency. The waves with frequencies below the lower hybrid 


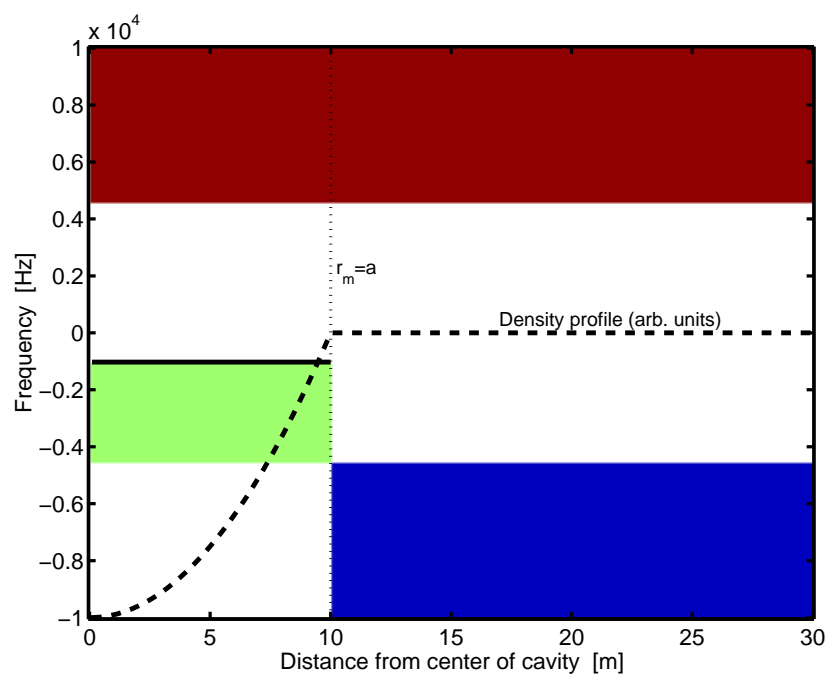

Fig. 1. The coloured areas give the regions where waves may exist (Eq. (26)). The cavity has in this example the parabolic shape used by Schuck et al. (1998), given in Eq. (25). Here we have used $\Delta=0.35, a=10 \mathrm{~m}, m=1$ and $k_{z}=0.003 \mathrm{~m}^{-1}$. We have also assumed the ions to be oxygen ions, and $f_{\mathrm{LH}}=4600 \mathrm{~Hz}$ and $f_{c e}=8 \times 10^{5} \mathrm{~Hz}$. Positive frequencies correspond to waves rotating in the right-handed direction with respect to the magnetic field, while negative frequencies indicate left-handed rotation. The solid black line is the limiting frequency, $\omega_{m} /(2 \pi)$, given by Eq. (27). The density profile is also included for comparison.

frequency had a discrete spectrum of eigenfrequencies, and above the lower hybrid frequency the eigenfrequencies were continuous. We will here consider the situation for a general shape, $v(r)$, of the density depletion.

\section{Existence of wave solutions}

The behaviour of the solutions of Eq. (24) depends on the sign of the function $A(r)$. In the regions where $A(r)$ is positive the radial component of the electric field has the general behaviour of Bessel functions, and in the regions where $A(r)$ is negative the behaviour is that of the modified Bessel functions (Watson, 1944). This means that we have wave solutions only when

$A(r)>0$

This condition gives the region where waves may exist, while the behaviour of individual eigenmodes within this region are discussed in the next section. The limiting case, where $A(r)=0$, is found for

$\omega_{m}=-\frac{k_{z}^{2} \omega_{c e} r}{m(d \nu / d r)}$.

Assuming that $d v / d r \geq 0$ for all $r$, we see that the condition given in Eq. (26) is satisfied for two intervals of $\omega$. The first interval is given by $0<\omega_{\mathrm{LH}}<\omega<\infty$, which means that we always have solutions with $|\omega|>\omega_{\mathrm{LH}}$, but no solutions with
$|\omega|<\omega_{\mathrm{LH}}$, that are rotating in a right-handed direction with respect to the magnetic field. The second interval is for values of $\omega$ between $-\omega_{\mathrm{LH}}$ and $\omega_{m}$, which means left-handed rotating waves. These waves will be examined closer.

In the following, we assume that $(d v / d r) / r$ is a decreasing function for all $r$, which is valid for many realistic cavities. Then, for a given $m$ and $k_{z}$, there is a specific value of the radius, $r_{m}$, where $\omega_{m}=-\omega_{\mathrm{LH}}$. This radius is given by

$\left.\frac{1}{r_{m}} \frac{d \nu}{d r}\right|_{r=r_{m}}=\frac{k_{z}^{2} \omega_{\mathrm{ce}}}{m \omega_{\mathrm{LH}}}$.

Considering propagating (non-evanescent) left-hand rotating waves, inside $r_{m}$ we have waves at frequencies $-\omega_{L H}<\omega<\omega_{m}<0$, while outside $r_{m}$ there are propagating waves for $\omega_{m}<\omega<-\omega_{\mathrm{LH}}<0$. This means that the waves with left-handed rotation around the magnetic field have $|\omega|<\omega_{\mathrm{LH}}$ inside $r_{m}$, and $|\omega|>\omega_{\mathrm{LH}}$ outside $r_{m}$. The radius $r_{m}$ may be used as the definition of the extension of the trapped wave field for a specific wave (given $k_{z}$ and $m$ ). Note that this definition is valid for a rather general shape of the cavity, and that the density depletion may be much wider than the region of wave trapping given by $r_{m}$. The wave electric field for frequencies below $\omega_{\mathrm{LH}}$ does not disappear outside the radius $r_{m}$, but it becomes exponentially smaller as $r \rightarrow \infty$.

As an example of the behavior of the waves, we consider the parabolic density profile (25). For the parameters used by Schuck et al. (1998) $(\Delta=0.35, a=10 \mathrm{~m}, m=1$, $\left.k_{z}=0.003 \mathrm{~m}^{-1}\right)$, the values of $\omega$ and $r$, where the condition in Eq. (26) is satisfied, are indicated in Fig. 1, together with the limiting frequency $\omega_{m}$. The radius $r_{m}$ inside which trapped eigenmodes are non-evanescent is marked in the figure. Three different wave regions are seen in Fig. 1. We have waves rotating in the right-handed direction with respect to the background magnetic field for all distances from the centre of the cavity, but only with frequencies above the ambient lower hybrid frequency. These waves are marked with red in Fig. 1. We have left-handed rotating waves above the lower hybrid frequency for large distances from the cavity centre, marked with blue. Finally, we have left-handed rotating waves below the lower hybrid frequency, marked with green, at distances up to $r_{m}=a$ from the centre of the cavity. We may note that the abrupt cut-off of all frequencies below $\omega_{\mathrm{LH}}$ at $r_{m}$ is a feature of the parabolic density profile, where $r_{m}$ equals $a$, the radius where the density depletion ends. This is not the case for other density profiles.

We now again consider a more general shape of the density cavity. Note that Eq. (28) gives a condition on the density gradient, concerning when the cavity can trap waves. When the condition

$\frac{d \nu}{d r}<\frac{k_{z}^{2} \omega_{\mathrm{ce}} r}{m \omega_{\mathrm{LH}}}=\left(\frac{k_{z}}{\tilde{k}_{\perp}}\right)^{2} \tilde{k}_{\perp} \frac{\omega_{\mathrm{ce}}}{\omega_{\mathrm{LH}}}$

is satisfied for all $r$, it is impossible to find a solution to Eq. (28). Here we have introduced the azimuthal wave number $\tilde{k}_{\perp}=m / r$. From the last part of Eq. (29) it is clear that, for a given ratio $k_{z} / \tilde{k}_{\perp}$, we must have a sharper density gradient 
for larger $\tilde{k}_{\perp}$ in order for the effects of the cavity to occur. For a parabolic density profile, as in Eq. (25), this condition turns into

$$
\frac{\Delta}{a^{2}}>\frac{k_{z}^{2} \omega_{\mathrm{ce}}}{2 m \omega_{\mathrm{LH}}}, \quad r<a,
$$

for trapped modes to be possible in the cavity. Thus, the radius $r_{m}$ always equals $a$, and Eq. (30) gives the condition for a specific wave (given $k_{z}$ and $m$ ) to be trapped.

The behaviour close to the center of the cavity, $r \rightarrow 0$, may also be studied for a rather general cavity shape using the function $A(r)$ given in Eq. (23). This function goes to a constant when $r \rightarrow 0$, which means that the first term in the coefficient in front of $\tilde{\varphi}$ in Eq. (24) is negligible compared to the second term. Thus, close to the center, we can write the solution to Eq. (24) as

$\tilde{\varphi}(r)=C r^{m}+\frac{D}{r^{m}}$,

where $C$ and $D$ are constants. For physical reasons, we must put $D=0$ in order to have a bounded solution when $r \rightarrow 0$, so we conclude that the solution must be of the form $\tilde{\varphi}(r)=C r^{m}$ close to the center of the cavity. The perpendicular components of the wave electric field is then given by $\boldsymbol{E}_{\perp}=-\nabla_{\perp} \varphi$, so that we have

$$
\left\{\begin{array}{l}
E_{r}=-C m r^{m-1} e^{i\left(m \theta+k_{z} z-\omega t\right)} \\
E_{\theta}=-i C m r^{m-1} e^{i\left(m \theta+k_{z} z-\omega t\right)}
\end{array} .\right.
$$

For $m>1$ the electric field vanishes at the center of the cavity and for $m=1$ it is finite with no curvature or gradient. There is thus no shear in the electric field at $r=0$.

The conclusion here is that inside $r_{m}$, we have waves with right-handed rotation with frequencies above the ambient lower hybrid frequency, and left-handed rotation below the lower hybrid frequency. Outside this radius, all nonevanescent waves have frequencies above the lower hybrid frequency and can be described as a mix of right- and lefthand rotating waves, i.e. as the linear waves in a homogeneous plasma. We have also found that there is a lower limit, given by Eq. (27), for the frequencies that may occur inside a density cavity. Furthermore, there is a condition that the density gradient must satisfy for waves with frequencies below the ambient lower hybrid frequency to occur. When we are sufficiently far from the center of the cavity, at a sufficiently small density gradient, the waves are unaffected by the cavity.

\section{Existence of discrete eigenmodes}

Above we investigated the general condition for trapped waves to be able to exist in a density cavity, Eq. (26). We are also interested in examining the conditions for the existence of a discrete spectrum of frequencies, i.e. the existence of discrete eigenmodes. For this purpose it is convenient to introduce a new function $\Phi(r)$, given by

$\Phi(r)=\sqrt{r} \tilde{\varphi}(r)$.
With this function, we can rewrite Eq. (24) as

$\frac{d^{2} \Phi}{d r^{2}}+\left(A(r)+\frac{1-4 m^{2}}{4 r^{2}}\right) \Phi=0$.

In order to further simplify the notation we introduce a generalized potential function

$V(r)=-\frac{\omega_{L H}^{2} m \omega}{\omega_{\mathrm{ci}}\left(\omega^{2}-\omega_{\mathrm{LH}}^{2}\right) r} \frac{d \nu}{d r}-\frac{1-4 m^{2}}{4 r^{2}}$,

and a generalized energy parameter

$E=\frac{m_{\mathrm{i}} \omega_{\mathrm{LH}}^{2} k_{z}^{2}}{m_{\mathrm{e}}\left(\omega^{2}-\omega_{\mathrm{LH}}^{2}\right)}$.

These definitions make it possible for us to rewrite Eq. (34) in a more familiar form, as

$-\frac{d^{2} \Phi}{d r^{2}}+(V(r)-E) \Phi=0$.

This has the form of a time-independent Schrödinger Equation, for which we know the behaviour of the solutions for different combinations of $V(r)$ and $E$ (see, for instance, Bransden and Joachain (1989)). Non-evanescent solutions in a cavity where $V(r) \rightarrow 0$ when $r \rightarrow \infty$ have a discrete spectrum of eigenvalues for the generalized energy, as long as $E<0$ and a continuous spectrum otherwise. It is clear from Eq. (36) that this condition for having a discrete spectrum is equivalent to $|\omega|<\omega_{\mathrm{LH}}$. The waves with frequencies below the lower hybrid frequency have a discrete spectrum of eigenfrequencies, while the waves above the lower hybrid frequency have a continuous spectrum, for given values of $m$ and $k_{z}$. This is in agreement with the results of Schuck et al. (1998), where the eigenfrequencies for a parabolic density depletion are found analytically, but for a general density profile these frequencies have to be found numerically. Section 4 only gives the possible values of the eigenfrequencies, but this is sufficient to know in most situations, since the eigenfrequencies often are close to each other (Schuck et al., 1998).

\section{Gaussian cavities}

It has been shown by Høymork et al. (2000) that the measured density profiles of the lower hybrid cavities agree well with a Gaussian shape. We may then write a typical profile of a density depletion as

$v(r)=-\Delta e^{-\frac{r^{2}}{b^{2}}}$,

where $\Delta>0$ is the depth of the cavity. In this case the function $A(r)$, defined in Eq. (23), is given by

$A(r)=\frac{m_{\mathrm{i}} \omega_{\mathrm{LH}}^{2}}{m_{\mathrm{e}}\left(\omega^{2}-\omega_{\mathrm{LH}}^{2}\right)}\left(k_{z}^{2}+\frac{2 m \Delta \omega}{\omega_{\mathrm{ce}} b^{2}} e^{-\frac{r^{2}}{b^{2}}}\right)$. 


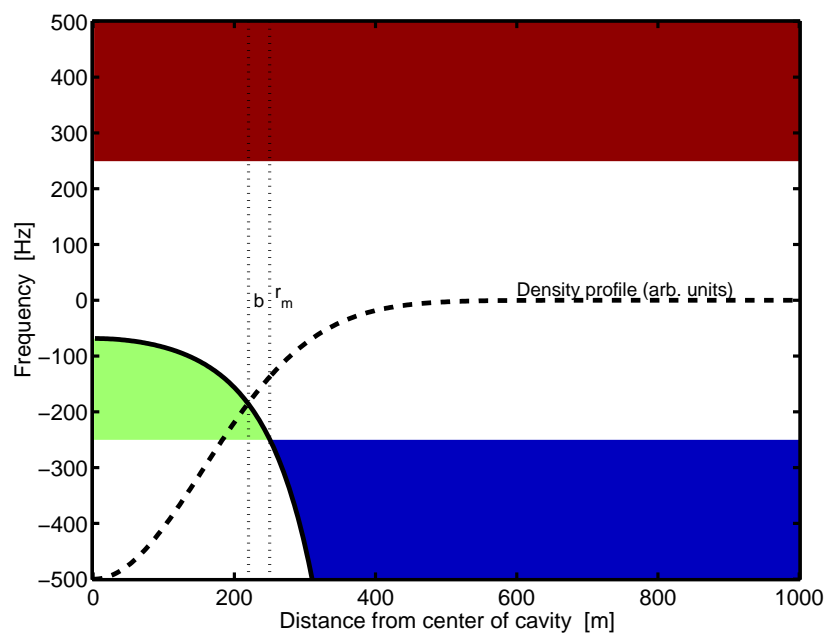

Fig. 2. The coloured areas show the wave frequencies we theoretically may find on different distances from the center of the cavity. In this example we have a density depletion with Gaussian shape as in Eq. (38), with $f_{\mathrm{ce}}=11 \mathrm{kHz}, f_{\mathrm{LH}}=250 \mathrm{~Hz}, \Delta=0.35, b=220 \mathrm{~m}$ and $k_{z}=3 \times 10^{-4} \mathrm{~m}^{-1}$. A positive frequency corresponds to waves rotating in the right-handed direction, while negative frequencies indicate left-handed rotation. The solid black line is the limiting frequency, $\omega_{m} /(2 \pi)$. The density profile is included for comparison.

The radius of a Gaussian cavity can then, according to Eq. (28), be written as

$r_{m}=b \sqrt{\ln \left(\frac{2 \Delta m \omega_{\mathrm{LH}}}{k_{z}^{2} \omega_{\mathrm{ce}} b^{2}}\right)}$.

In order for this depletion to be able to have wave modes below the ambient lower hybrid frequency, we must have a real value of $r_{m}$. This gives a condition for the geometry of the Gaussian cavity

$\frac{\Delta}{b^{2}}>\frac{k_{z}^{2}}{2 m} \frac{\omega_{\mathrm{ce}}}{\omega_{\mathrm{LH}}}$,

that must be satisfied in the cavity if waves below $\omega_{\text {LH }}$ are to be trapped. This condition is the same condition as we find for the parabolic density profile (25), but in that case the parameter $b$ is changed to $a$, the radius of the cavity. Equation (41) can also be interpreted as giving the maximum possible value of $k_{z}$ for a trapped mode, when the geometry of the cavity is given, which has also been noticed by Schuck (1999) in the case of parabolic density profile. In Sect. 7 we use Freja data to statistically estimate the maximum possible $k_{z}$ for a set of observed LHCs.

In order to find a parabolic density profile (25) that approximates a Gaussian profile (38), we put some restrictions on that profile. We want the profile to have the same total number of particles depleted and in addition one possibility is to choose the same density depth in the center. These conditions yield that a Gaussian cavity (38) is best approximated by a parabolic cavity of the same depth and with radius $\sqrt{2} b$, which can be found by straightforward integration.
As an example of a cavity with a Gaussian density profile, we consider a hydrogen plasma with an electron cyclotron frequency of $11 \mathrm{kHz}$ and a lower hybrid frequency of $250 \mathrm{~Hz}$ outside the cavity. These are parameters taken from the first LHC observation on Cluster (Tjulin et al., 2003). The density depletion is taken to have $\Delta=0.35$ and $b=220 \mathrm{~m}$, and $k_{z}$ is assumed to be $3 \times 10^{-4} \mathrm{~m}^{-1}$. We may note that the observed projected perpendicular wave number for this event was about $5 \times 10^{-3} \mathrm{~m}^{-1}$, so the assumed value of $k_{z}$ satisfies the condition $k_{z} \ll k_{\perp}$ that was used in the derivations. For $m=1$ the values of $\omega$ and $r$, where the condition in Eq. (26) is satisfied, are indicated in Fig. 2.

The same wave regions as in Fig. 1 are seen in Fig. 2. The maximum distance from the center of the cavity where the waves may have frequencies below the ambient lower hybrid frequency is about $320 \mathrm{~m}$ in this example. Outside this radius, the density gradient is too weak for the existence of trapped eigenmodes. The lowest possible wave frequency inside the cavity is about $68 \mathrm{~Hz}$, which is only about $25 \%$ of the ambient lower hybrid frequency. The local lower hybrid frequency at the cavity centre is about $245 \mathrm{~Hz}$ in this example, so the frequency shift is mostly due to effects from the density gradient, and not the change in local lower hybrid frequency. The lowest possible actual frequency, that is the lowest frequency in the discrete frequency spectrum, can be simply determined only for a parabolic cavity but numerical investigations can be made for all reasonably smooth cavity shapes. However, the lowest measured frequency inside the cavity was about $70 \mathrm{~Hz}$, consistent with our prediction in Fig. 2. Note that the prediction depends on the assumed value of $k_{z}$. In Sect. 8 we use the Cluster event discussed here and the limiting case of Eq. (26) to estimate $k_{z}$ for an LHC.

\section{Application to Freja statistics}

In the following we use the shape of the density depletion of several LHCs observed during a Freja satellite crossing of the auroral region to estimate the parallel wave number of the waves in the cavities. The data in Fig. 3 are taken from Høymork et al. (2000) and show the widths and depths of the cavities. We have superimposed contours of maximum $k_{z}$ (in units of $\mathrm{m}^{-1}$ ), for different $b(1 / e$-width) and $\Delta$ (relative depth), from relation (41). We have used $m=1$, and the typical values $\omega_{\mathrm{LH}} /(2 \pi)=2100 \mathrm{~Hz}$ and $\omega_{\mathrm{ce}} /(2 \pi)=8.4 \times 10^{5} \mathrm{~Hz}$. Since the spacecraft usually does not pass the center of the cavities, the measured depths in Fig. 3 are smaller than the actual depths of the cavities, which results in an underestimate of the maximum $k_{z}$. The estimates of the cavity widths are however independent of how far from center of the cavity the spacecraft passes since the cavity are assumed to be Gaussian. This means that the error bars for the measurements in Fig. 3 is one-sided, pointing towards larger depth. By the the distribution of the measurements it seems unlikely that the typical cavity corresponds to a value of $k_{z}$ larger than about $0.001 \mathrm{~m}^{-1}$, corresponding to a minimum parallel 


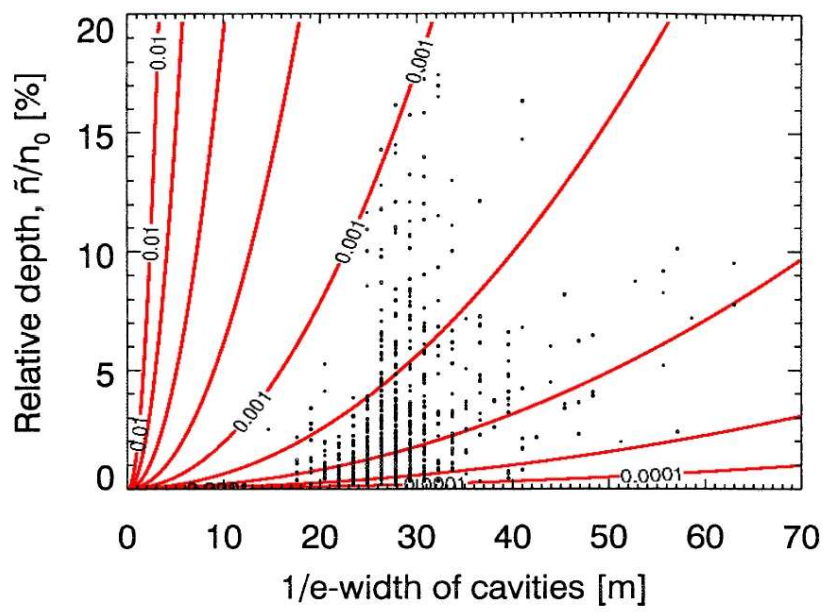

Fig. 3. Scatter plot of the measured $1 / e$-widths and depths of 672 cavities from orbit 1234 of the Freja satellite. Data are taken from Høymork et al. (2000). We have superimposed contours of the maximum values of $k_{z}$ (unit $\mathrm{m}^{-1}$ ) in red, for given width and depth of a cavity according to Eq. (41). The parameters used are $m=1, f_{L H}=2100 \mathrm{~Hz}$ and $f_{\mathrm{ce}}=8.4 \times 10^{5} \mathrm{~Hz}$. The relative depth corresponds to the parameter $\Delta$ in Eq. (41) and the $1 / e$-width to $b$ in the same equation. Underlying dataplot reproduced by permission of the American Geophysical Union.

wavelength of the order of $6 \mathrm{~km}$. By assuming that the length of the cavity along the magnetic field is at least one parallel wavelength, we get an estimate of the minimum cavity length for these observations to be $6 \mathrm{~km}$. This can be compared with the measured $1 / e$-widths of the cavities of about $30 \mathrm{~m}$. The LHCs are indeed elongated structures.

\section{Application to Cluster data}

Let us now examine what may be investigated by a spacecraft that is passing a cavity, as in Fig. 4, where $r_{0}$ is the closest approach to the center of the cavity. Let $x=v_{\mathrm{sc}} t$ be the distance travelled by the spacecraft, with $t=0$ at the closest approach and $v_{s c}$ is the spacecraft velocity. For a Gaussian density profile, as in Eq. (38), the measured density time series is

$\nu(t)=-\Delta e^{-\frac{r_{0}^{2}+x(t)^{2}}{b^{2}}}=-\Delta e^{-\frac{r_{0}^{2}}{b^{2}}} e^{-\frac{\left(v_{s c t}\right)^{2}}{b^{2}}}$,

and it is seen that the $1 / e$-width of the density cavity as measured by the spacecraft is identical to the width $b$ in the density profile. The measured depth, however, differs by a factor $\exp \left(-r_{0}^{2} / b^{2}\right)$ from the actual depth.

The lowest angular frequency that can possibly be found inside the cavity is given by $\left|\omega_{m}\right|$ from Eq. (27). As was noted in Sect. 5, the discreteness of the spectrum may mean that no wave mode can exist at this frequency, but the eigenfrequencies found by Schuck et al. (1998) indicates that modes will exist sufficiently close above this value to give a reasonable estimate of the lowest frequency, assuming that $m=1$. It is then possible to fit the lowest measured frequency

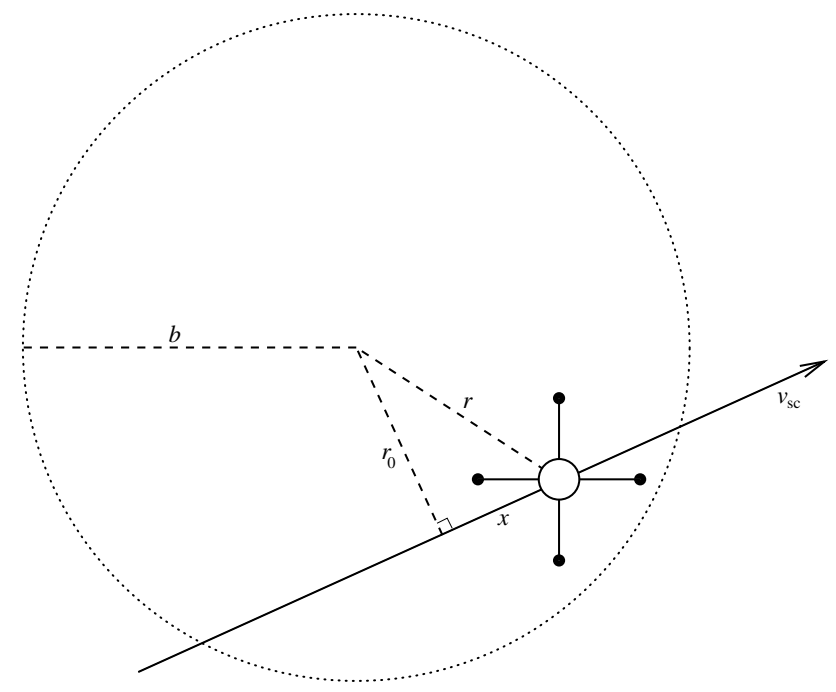

Fig. 4. The geometry of a spacecraft passing a lower hybrid cavity. The distance $r_{0}$ is the closest approach to the cavity center, and $x=v_{s c} t$ is the distance from the point of closest approach along the spacecraft trajectory.

with Eq. (27) to obtain an estimate of the parameters $r_{0}, \Delta$ and $k_{z}$. Thus, we may find important parameters for the cavity by measuring the density profile and especially the profile of the lowest frequency, which usually cannot be well determined, for example, since the spacecraft spends only a short time inside the LHC and it is not clear how stationary the emissions inside the cavity are. The parallel wavelength is given by $2 \pi / k_{z}$, and should be of the size, or smaller, than the length of the cavity. We may hence use $k_{z}$ to obtain a lower bound on the length of the cavity.

An attempt to estimate $k_{z}$ has been done using data from the Cluster event analysed by Tjulin et al. (2003). This is yet the only LHC event found in the Cluster EFW data, where the instrument sampling frequency is sufficient for this kind of analysis. The results are shown in Fig. 5, where the upper panel shows the plasma density, determined by using the spacecraft potential (Pedersen et al., 2001) as measured by the EFW instrument (Gustafsson et al., 1997). By fitting a Gaussian to the data, we find $b=220 \mathrm{~m}$. The lower panel shows a wavelet spectrogram of the duskward electric field, measured by EFW. The line is a fit of $\left|\omega_{m}\right|$, from Eqs. (27) and (42), to the lowest frequency in the spectrogram, using a nonlinear fitting algorithm, with the resulting parameters $k_{z}=2.5 \times 10^{-4} \mathrm{~m}^{-1}, \Delta=0.33$ and $r_{0}=73 \mathrm{~m}$. The parallel wavelength for the waves in this cavity is then about $25 \mathrm{~km}$, giving an estimate of the length of the cavity of at least $25 \mathrm{~km}$. The parameters we found are at least reasonable, since the maximum possible value of $k_{z}$ for this geometry, from Eq. (41), is about $5 \times 10^{-4} \mathrm{~m}^{-1}$, but, as mentioned above, the errors may be large. A statistical estimate using several LHCs would increase the reliability. 

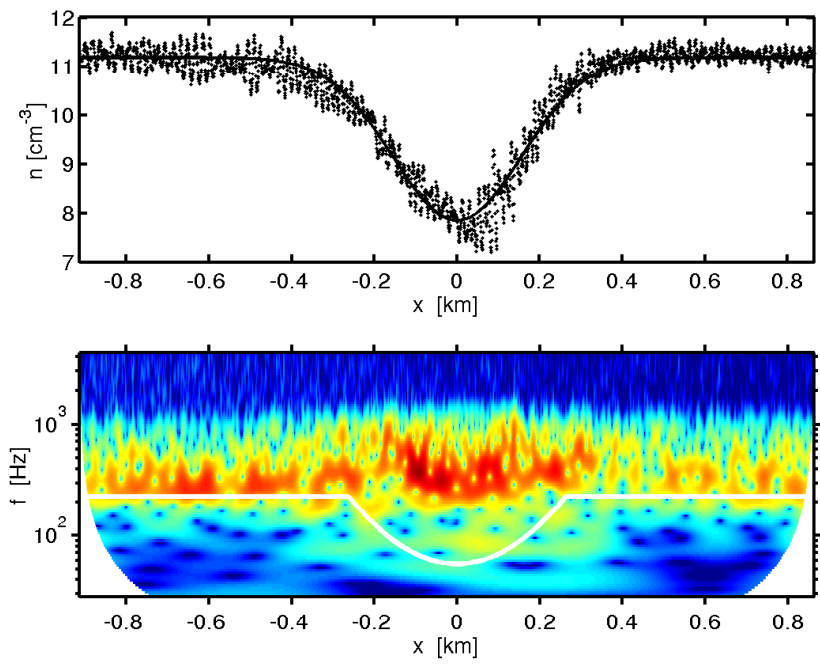

Fig. 5. An example on how to apply the theory to measured data, in this case the Cluster event analyzed by Tjulin et al. (2003). The dots in the upper panel is the density, derived from the spacecraft potential from the EFW instrument, and the white line is the fitted Gaussian $(b=220 \mathrm{~m})$. The lower panel is a wavelet spectrogram of the duskward electric field. The solid line is a fit to the lowest frequency in the spectrogram. We have $k_{z}=2.5 \times 10^{-4} \mathrm{~m}^{-1}, \Delta=0.33$ and $r_{0}=73 \mathrm{~m}$ for this fit.

\section{Discussion and conclusions}

We have in Sect. 7 examined some statistics from the Freja satellite, and found a maximum possible value of $k_{z}$ to be about $10^{-3} \mathrm{~m}^{-1}$. Typical $1 / e$-widths, $b$, of the cavities were about $30 \mathrm{~m}$. In Sect. 8 we have examined one cavity observed by one Cluster spacecraft, and obtained a value of $k_{z}$ of about $2.5 \times 10^{-4} \mathrm{~m}^{-1}$ for $b=220 \mathrm{~m}$. The ratio between the parallel and the perpendicular scale lengths of the cavities thus seems to be between 100 and 200 for these data sets. Indirect arguments from the signatures of transversely accelerated ions led Vago et al. (1992) to assume that the parallel extension of LHCs was "probably a large fraction" of the $700-1100 \mathrm{~km}$ altitude segment investigated by the TOPAZ II sounding rocket. Direct observations from the OEDIPUS-C sounding rocket (Knudsen et al., 1999) showed a lower limit of the parallel length of the LHCs to be $820 \mathrm{~m}$ at an altitude of $670 \mathrm{~km}$. Our estimates are in line with those observations.

To review the relevance of our results from cold plasma theory, we reconsider the electrostatic dispersion relation for lower hybrid waves in a warm homogeneous plasma, as described by Eq. (1). As $k_{z} \rightarrow 0$, the thermal effects become more important in the homogeneous case, at least close to the lower hybrid frequency, so we expect that this is also the case in our inhomogeneous plasma. On the other hand, since Schuck and Bonnell (2003) have shown that the Hall correction (the shift in frequency from the density gradient) is more important than the thermal effects for lower hybrid cavities, we expect that our results should also hold when thermal effects are included, at least for trapped modes well below $\omega_{\mathrm{LH}}$. As our estimates of $k_{z}$ are derived for such trapped modes we still expect them to be reasonable. The electromagnetic effects are important to include for examining how the waves inside the density cavity couple to magnetosonic waves outside the cavity, but they should not be important for the features of the mainly electrostatic waves that we have studied.

Our conclusions are:

- The results of Schuck et al. (1998) for a parabolic shape of the density depletion are also valid for more general density profiles. The waves inside the cavity, with frequencies above the ambient lower hybrid frequency, have a continuous frequency spectrum and are rotating in a right-handed fashion with respect to the magnetic field, while the waves with frequencies below the lower hybrid frequency have a discrete frequency spectrum, for given $k_{z}$, and rotate in a left-handed fashion. These results also follow from numerical simulations using a smoothed parabolic density profile (Schuck et al. 1998), a profile that satisfies the conditions for the results in this paper to be valid. It may also be noted that the ray-tracing analysis performed by Schuck and Bonnell (2003) used a cigar shaped, three-dimensional Gaussian density depletion.

- Even though the density depletion may go to zero slowly with increasing $r$, and thus be very large in extent, there is a largest distance, $r_{m}$, within which a trapped wave, with given $k_{z}$ and azimuthal mode number $m$, may propagate. This distance is given by Eq. (28), and it may be taken to define the width of the wave field for a certain wave mode.

- From Eq. (28) also follows that there is a lower limit on the density gradient of the cavity for rotating waves with $|\omega| \ll \omega_{\mathrm{LH}}$ to occur at all. For given limits on $k_{z}$ and $m$, this can be used to define which density cavities should be regarded as potential LHCs, as opposed to density depletions without trapped waves.

- There is a lower limit to the frequencies that can exist in a density cavity, and there is a local relation between the plasma density gradient and the lowest possible frequency.

- Putting our findings together, we combine theory with spacecraft observations along approximately perpendicular crossings of LHCs to estimate the parallel wavenumber $k_{z}$ of the cavities. This also gives an estimate of the length of a typical cavity along the magnetic field. Our examples indicate that the length of the cavities is larger than the width by a factor of at least 100 .

Acknowledgements. Thanks to J.-O. Hall for enlightening discussions.

Topical Editor T. Pulkkinen thanks P. Schuck and K. Lynch for their help in evaluating this paper. 


\section{References}

Bonnell, J. W., Schuck, P. W., Pinçon, J.-L., Seyler, C. E., and Kintner, P. M.: Observation of bound states and counterrotating lower hybrid eigenmodes in the auroral ionosphere, Phys. Rev. Lett., 80, 5734-5737, 1998.

Bransden, B. H. and Joachain, C. J.: Introduction to quantum mechanics, Longman Scientific \& Technical, Harlow, England, 1989.

Dovner, P. O., Eriksson, A. I., Boström, R., Holback, B., Waldemark, J., Eliasson, L., and Boehm, M.: The occurence of lower hybrid cavities in the upper ionosphere, Geophys. Res. Lett., 24, 619-622, 1997.

Eriksson, A. I., Holback, B., Dovner, P. O., Boström, R., Holmgren, G., André, M., Eliasson, L., and Kintner, P. M.: Freja observations of correlated small-scale density depletions and enhanced lower hybrid waves, Geophys. Res. Lett., 21, 1843-1846, 1994.

Gustafsson, G., Boström, R., Holback, B., Holmgren, G., Lundgren, A., Stasiewicz, K., Åhlén, L., Mozer, F. S., Pankow, D., Harvey, P., Berg, P., Ulrich, R., Pedersen, A., Schmidt, R., Butler, A., Fransen, A. W. C., Klinge, D., Thomsen, M., Fälthammar, C.G., Lindqvist, P.-A., Christenson, S., Holtet, J., Lybekk, B., Sten, T. A., Tanskanen, P., Lappalainen, K., and Wygant, J.: The electric field and wave experiment for the Cluster mission, Space Sci. Rev., 79, 137-156, 1997.

Hall, J. O., Eriksson, A. I., and Leyser, T. B.: Excitation of localized rotating waves in plasma density cavities by scattering of fast magnetosonic waves, Phys. Rev. Lett., 92, doi:10.1103/PhysRevLett.92.255002, 2004.

Høymork, S. H., Pécseli, H. L., Lybekk, B., Trulsen, J., and Eriksson, A. I.: Cavitation of lower hybrid waves in the Earth's ionosphere: A model analysis, J. Geophys. Res., 105, 18 519-18 535, 2000.

Kjus, S. H., Pécseli, H. L., Lybekk, B., Holtet, J., Trulsen, J., Lühr, H., and Eriksson, A.: Statistics of the lower hybrid wave cavities detected by the Freja satellite, J. Geophys. Res., 103, 26633$26647,1998$.

Knudsen, D. J., Wallis, D. D., and James, H. G.: Tethered two-point measurements of solitary auroral density cavities, Geophys. Res. Lett., 26, 2933-2936, 1999.

Lynch, K. A., Arnoldy, R. L., Kintner, P. M., Schuck, P., Bonnell, J. W., and Coffey, V.: Auroral acceleration from lower hybrid solitary structures: A summary of sounding rocket observations, J. Geophys. Res., 104, 28 515-28 534, 1999.
Pécseli, H. L., Iranpour, K., Holter, Ø., Lybekk, B., Holtet, J., Trulsen, J., Eriksson, A. I., and Holback, B.: Lower-hybrid cavities detected by the Freja satellite, J. Geophys. Res., 101, 52995316, 1996.

Pedersen, A., Décréau, P., Escoubet, C.-P., Gustafsson, G., Laakso, H., Lindqvist, P.-A., Lybekk, B., Masson, A., Mozer, F., and Vaivads, A.: Four-point high time resolution information on electron densities by the electric field experiments (EFW) on Cluster, Ann. Geophys., 19, 1483-1489, 2001.

Pinçon, J.-L., Kintner, P. M., Schuck, P., and Seyler, C. E.: Observation and analysis of lower hybrid solitary structures as rotating eigenmodes, J. Geophys. Res., 102, 17 283-17 296, 1997.

Schuck, P. W.: A theory of lower hybrid solitary structures: Rotating helical eigenstates excited by wave scattering, Ph.D. thesis, Cornell University, Ithaca, NY, 1999.

Schuck, P. W. and Bonnell, J. W.: Ray trajectories of lower hybrid solitary structures, J. Geophys. Res., 108, 1175, doi:10.1029/2002JA009, 716, 2003.

Schuck, P. W., Seyler, C. E., Pinçon, J.-L., Bonnell, J. W., and Kintner, P. M.: Theory, simulation and observation of discrete eigenmodes associated with lower hybrid solitary structures, J. Geophys. Res., 103, 6935-6953, 1998.

Schuck, P. W., Ganguli, G. I., and Kintner, P. M.: The role of lowerhybrid-wave collapse in the auroral ionosphere, Phys. Rev. Lett. 89, doi:10.1103/PhysRevLett.89.065 002, 2002.

Schuck, P. W., Bonnell, J. W., and Kintner, JR, P. M.: A review of lower hybrid solitary structures, IEEE Transactions on Plasma Science, 31, 1125-1177, 2003.

Seyler, C. E.: Lower hybrid wave phenomena associated with density depletions, J. Geophys. Res., 99, 19513-19525, 1994.

Shapiro, V. D., Shevchenko, V. I., Solov'ev, G. I., Kalinin, V. P., Bingham, R., Sagdeev, R. Z., Ashour-Abdalla, M., Dawson, J., and $\mathrm{Su}$, J. J.: Wave collapse at the lower-hybrid resonance, Phys. Fluids B, 5, 3148-3162, 1993.

Tjulin, A., Eriksson, A. I., and André, M.: Lower hybrid cavities in the inner magnetosphere, Geophys. Res. Lett., 30, 1364, doi:10.1029/2003GL016915, 2003.

Vago, J. L., Kintner, P. M., Chesney, S. W., Arnoly, R. L., Lynch, K. A., Moore, T. E., and Pollock, C. J.: Transverse ion acceleration by localized lower hybrid waves in the topside auroral ionosphere, J. Geophys. Res., 97, 16 935-16957, 1992.

Watson, G. N.: A treatise on the theory of Bessel functions, Cambridge University Press, Cambridge, 2nd ed., 1944. 